\title{
Real-Time Robust Video Stabilization Based on Empirical Mode Decomposition and Multiple Evaluation Criteria
}

\author{
Jun $\mathrm{Yu}^{(\bowtie)}$, Chang-wei Luo, Chen Jiang, Rui Li, Ling-yan Li, \\ and Zeng-fu Wang \\ Deptartment of Automation, University of Science and Technology of China, \\ Hefei 230026, China \\ \{harrtjun, zfwang\}@ustc.edu.cn
}

\begin{abstract}
A real-time robust video stabilization system is proposed. Firstly, SIFT feature points are extracted and matched between the reference frame and current frame, and then global motion parameters are obtained by fitting the feature matches with random sample consensus algorithm. Secondly, multiple evaluation criteria, i.e., global motion parameters and location errors of corresponding feature matches, are fused by empirical mode decomposition to smooth global motion for obtaining correction vector. Thirdly, motion compensation is applied to the current frame by using correction vector. Finally, stabilized video is obtained after each frame is completed by combining the texture synthesis method and the spatio-temporal information of video. By comparing the jittered video and stabilized video, the experimental results demonstrate the system can increase the average peak signal-to-noise ratio around $7.2 \mathrm{~dB}$, the identification ability and perceptive comfort on video content.
\end{abstract}

Keywords: Global motion estimation - Motion filtering and compensation · Video completion

\section{Introduction}

Video enhancement has been gaining in importance with the increasing prevalence of digital media. One of the most important enhancements is video stabilization [1]. Often, home videos suffer from an amount of unexpected image motion caused by unintentional shake of a human hand. The goal of video stabilization is to generate a new compensated video sequence where undesirable image motion is removed $[2,3]$.

\subsection{Related Works}

There are several major stages constituting a video stabilization process: global motion estimation [4], motion filtering and compensation [5], video completion [6].

Global motion estimation is achieved by estimating the interframe motion of adjacent frames [7]. In [8], global motion estimation using local motion vectors of 
subimages obtained by block-matching was presented. Motion estimation based on edge pattern matching was demonstrated in [9]. An iterative, multi-resolution motion estimation scheme that estimates affine motion parameters between levels of the Laplacian pyramid was described in [10, 11]. Fast motion estimation based on bit-plane matching was demonstrated in [12, 13]. A robust approach for phase correlation-based global motion estimation was demonstrated in [14]. Feature-based algorithms estimate interframe motion using extracted feature locations and robust filters $[15,16]$, these methods have gained larger consensus for their good performances [7, 17, 18].

In motion filtering, the estimated motion is evaluated to recognize intentional movement. Several techniques, such as DFT filtered frame position smoothing [14], Kalman filtering [19] and motion vector integration [20], were proposed and modified to correct translational and rotational jitters $[21,22]$ according to real systems constraints. After motion filtering, the jitter is obtained, then the image frames are spatially displaced inversely to jitter. This process is referred to as the motion compensation, and the compensation amount of each frame is referred to as the correction vector.

Filling in missing image areas in a video is called video completion [23]. In [24], mosaicing is used to fill up the missing image areas in the context of video stabilization. Wexler et al. [25] filled in the holes in a video by sampling spatio-temporal volume patches from different portions of the same video. Jia et al. [26] and Cheung et al. [27] proposed an approach by segmenting the video into a moving object layer and a static background layer. Cheung et al. [28] showed an effective video fill-in result in their video epitomes framework. Matsushita et al. [29] conduct the video completion based on the local motion estimation of missing image areas. Criminisi et al. [30] and Tang et al. [31] proposed a texture synthesis method. The most similar texture patch is searched around the missing image area to replace the unknown part in the original texture patch. It can achieve good result if enough similar information are available.

\subsection{Paper Contribution}

This paper proposes a real-time robust video stabilization system. Our work has following advantages: (1) Global motion is estimated by random sample consensus (RANSAC) fitting on the matched scale invariant feature transform (SIFT) features. (2) The advantage of empirical mode decomposition (EMD) is firstly verified in the video stabilization community, and a satisfying motion filtering result is obtained. (3) Multiple evaluation criteria are fused to increase the robustness of motion filtering and compensation, and thus the correction vector, especially the rotation component, can be obtained accurately in the motion filtering process, and used accurately in the motion compensation process. (4) Different from the single image, there are plenty of interframe similar information among the adjacent frames of video, Therefore, the image texture synthesis method in [30] is combined with the spatio-tempral information of video to carry out the video completion. 


\section{Framework}

Figure 1 is the framework of the proposed system. Firstly, when input jittered video, SIFT keypoints are extracted from two consecutive frames, and these two sets of features are matched and then fitted to obtain global motion parameters by the RANSAC algorithm. Secondly, by fusing multiple evaluation criteria, EMD is used to filter global motion for obtaining correction vector. Thirdly, according to evaluation criteria, motion compensation is applied to the current frame by using correction vector. Finally, missing image areas in each frame is completed by combining the texture synthesis method and the spatio-temporal information of video.

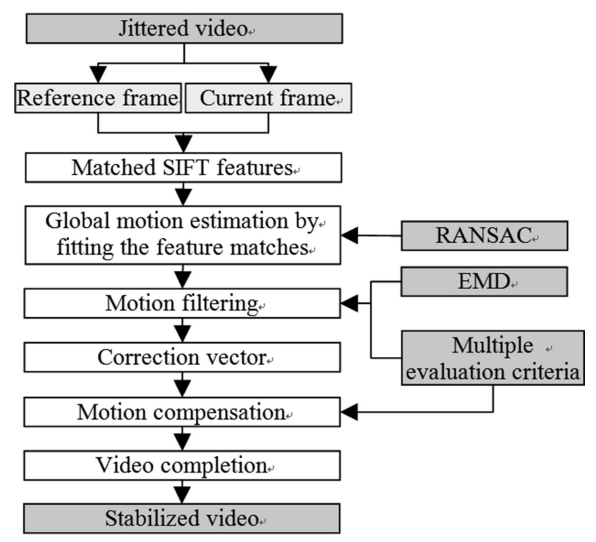

Fig. 1. Framework.

\section{Image Matching}

SIFT is designed for extracting highly distinctive invariant features from images [32, 33], which can be used to perform reliable matching of the same object between different images. SIFT algorithm has several free parameters, so our implementation is designed to make features more suitable for video stabilization. The first stage is building the scale-space pyramid. Two successive Gaussians are separated by a factor of $\sqrt{2}$ and 5 images per octave are computed, resulting in 4 Difference-of-Gaussian images and therefore extrema are searched on two different images. When keypoints are located, each of them is interpolated, evaluated for contrast and then passed through a Harris edge detector. Avoiding unstable features is very important in video stabilization. In order to obtain better features, original thresholds used in the SIFT algorithm for keypoints validation are hardened to discard a larger number of features. After orientation assignment has been fulfilled, a 128-element feature vector is derived and bilinear interpolation is successfully used to increase descriptor robustness and invariance. The final output is therefore a set of keypoints with their descriptors. 
The keypoint matching is performed using Euclidean distance between descriptors' vectors and a distance ratio, namely ratio of closest neighbor distance to that of the second-closest one, that can be checked against a threshold to discard false matches. In fact, correct matches should have lower ratios while wrong ones should have ratios closer to one. We investigated correlation between distance ratio and correctness of matches and found that using a value of 0.6 as threshold performs well in discarding wrong matches. The result of this matching process is a list of keypoints pairs that can be easily used as input of the feature-based motion estimation algorithm.

\section{Global Motion Estimation}

The set of features retrieved during features matching are used to estimate the motion needed to overlay the current frame on the previous frame to minimize the visible motion. Because not all matches give correct information about how the frame has moved relatively to the previous, it is necessary to discard wrong matches that do not fit into the estimated transformation and may mislead the process. Assuming the imaging model is a perspective projection model, the global motion between frames, associating feature $\left(x_{i}, y_{i}\right)^{T}$ in frame $I_{n}$ with feature $\left(x_{j}, y_{j}\right)^{T}$ in frame $I_{n+1}$, can be described by the following transformation:

$$
\begin{aligned}
& x_{j}=\left(a_{1} x_{i}+a_{2} y_{i}+a_{3}\right) /\left(a_{7} x_{i}+a_{8} y_{i}+1\right) \\
& y_{j}=\left(a_{4} x_{i}+a_{5} y_{i}+a_{6}\right) /\left(a_{7} x_{i}+a_{8} y_{i}+1\right)
\end{aligned}
$$

Where $\left(a_{1}, a_{2}, a_{3}, a_{4}, a_{5}, a_{6}, a_{7}, a_{8}\right)$ are the parameters to be solved. In order to estimate eight parameters, only four couples of features are needed. However, features may be affected by noise, so it is useful to apply a Levenberg-Marquardt (LM) method on a set of redundant equations.

The whole set of feature matches does not contain useful information for effective motion compensation, as probably it includes wrong matches or correct matches that indeed belong to self-moving objects in the filmed scene. Obviously there are some correct pairs that do represent real camera shakes but several points simply do not relate to such information. Here we use RANSAC [34] to deal with this problem. RANSAC is an iterative method to estimate parameters of a mathematical model from a set of observed data which contains outliers.

Firstly, six couples of features are selected randomly from the feature set $\boldsymbol{P}=\left\{\left(\left(x_{i}, y_{i}\right)^{T} ;\left(x_{j}, y_{j}\right)^{T}\right)\right\}, i, j=1 \cdots n$. These features are comprised of a subset $\boldsymbol{S}_{1}$ of $\boldsymbol{P}$, and a solution $\boldsymbol{M}_{1}$ is obtained from $\boldsymbol{S}_{1}$. Then a new subset $\boldsymbol{S}_{1}^{*}$ of $\boldsymbol{P}$ is obtained by $\boldsymbol{S}_{1}^{*}=\left\{\left(\left(x_{i}, y_{i}\right)^{T} ;\left(x_{j}, y_{j}\right)^{T}\right) \mid\left\|\begin{array}{l}x_{j}-\left(a_{1} x_{i}+a_{2} y_{i}+a_{3}\right) /\left(a_{7} x_{i}+a_{8} y_{i}+1\right) \\ y_{j}-\left(a_{4} x_{i}+a_{5} y_{i}+a_{6}\right) /\left(a_{7} x_{i}+a_{8} y_{i}+1\right)\end{array}\right\| \leq T\right\}, T$ is a given threshold.

Secondly, above process is repeated $K$ times, and the $S_{k}^{*}$ with the most elements is selected. $K$ is determined according to the criterion in [18].

Finally, LM method is applied on $\boldsymbol{S}_{k}^{*}$ to obtain the final solution $\boldsymbol{M}$. 


\section{Motion Filtering and Compensation}

EMD and multiple evaluation criteria are used in the motion filtering, and then multiple evaluation criteria are also used in the motion compensation.

\subsection{Motion Filtering}

The EMD method [35] can decompose any complicated data set into a finite and often small number of components. These components form a complete and nearly orthogonal basis for the original signal. Essentially, It is a multi-level de-noising process for signal, and can be described as intrinsic mode functions (IMF). Without leaving the time domain, EMD is adaptive and highly efficient. Since the decomposition is based on the local characteristic time scale of the data, it can be applied to nonlinear and nonstationary processes. Because the excellent de-noising characteristics, EMD has been widely used in the signal de-noising, is also used for motion filtering here.

Firstly, the estimated global motion parameters $\boldsymbol{M}$ is chosen as one criterion to evaluate the video jitter. EMD is applied on $\boldsymbol{M}$, and the smoothing components are set as the motion filtering result. However, we found the motion filtering result of $\boldsymbol{M}$ is not satisfying when the jitter has very frequent tiny rotation component. The reason is: when the rotation component is very tiny, the filtering result is almost same to the original value, thus the compensation effect is very limited, and the human visual system still feel jittery when watching the compensated result. To alleviate this problem, an additional criterion should be chosen. Here we use the Euclidean distance of matched keypoints (EDMK) between adjacent frames as the second criterion, and the EMD is also used to smooth the $x$ component and $y$ component of EDMK.

Finally, the average of the filtering results by both criteria is set as the final result.

\subsection{Motion Compensation}

Firstly, the correction vector for the first criterion is obtained by computing the difference between original parameters $\boldsymbol{M}$ and filtering parameters $\hat{\boldsymbol{M}}$. Then the motion compensation is applied by the correction vector as follows:

$$
\begin{aligned}
& x_{j}=\left(a_{1 c} x_{i}+a_{2 c} y_{i}+a_{3 c}\right) /\left(a_{7 c} x_{i}+a_{8 c} y_{i}+1\right) \\
& y_{j}=\left(a_{4 c} x_{i}+a_{5 c} y_{i}+a_{6 c}\right) /\left(a_{7 c} x_{i}+a_{8 c} y_{i}+1\right)
\end{aligned}
$$

Where $\left(a_{1 c}, a_{2 c}, a_{3 c}, a_{4 c}, a_{5 c}, a_{6 c}, a_{7 c}, a_{8 c}\right)$ is the correction vector, $\left(x_{i}, y_{i}\right)$ is the pixel position before compensation, $\left(x_{j}, y_{j}\right)$ is the pixel position after compensation.

Secondly, the correction vector for the second criterion is obtained by computing the difference between original EDMK and filtering EDMK. Then the motion compensation is applied by the correction vector as follows: 


$$
x_{j}=x_{i}+x_{c} \quad y_{j}=y_{i}+y_{c}
$$

Where $\left(x_{c}, y_{c}\right)$ is the correction vector, $\left(x_{i}, y_{i}\right)$ is the pixel position before compensation, $\left(x_{j}, y_{j}\right)$ is the pixel position after compensation.

Finally, the coordinates of pixels are set as the average coordinates of the pixels compensated by the first evaluation criterion and the pixels compensated by the second evaluation criterion.

In addition, when features are tracked through consecutive frames, the relative error is cumulated, and the compensation process would drift by the cumulative error. To solve this problem, a re-initialization control strategy is adopted. The cumulative error of original image sequence updated to current frame (cumulative error 1 ) and the cumulative error of stabilized image sequence updated to current frame (cumulative error 2) are computed respectively. If the absolute difference between cumulative error 1 and cumulative error 2 is below a given threshold, the previous frame of stabilized image sequence is used as the reference frame, otherwise, the previous frame of original image sequence is used as the reference frame.

\section{Video Completion}

As stated before, the texture synthesis method [30, 31] searches the most similar texture patch around the missing image area to replace the unknown part in the original texture patch. However, because the similar information in the single image is usually not enough, it is hard to obtain satisfying result only by this method. In other word, there are plenty of interframe similar information among the adjacent frames of video. Therefore, the texture synthesis method can be improved if the interframe information is introduced. The way of combining them is: the most similar texture patch of an original texture patch $\boldsymbol{A}$ in the current frame is searched in the adjacent frames by the texture synthesis method [30]. If it is found, and the found texture patch is $\boldsymbol{B}$ in the adjacent frames, the neighbor texture patch of $\boldsymbol{B}$ will have the high priority to be the most similar texture patch of the neighbor texture patch of $\boldsymbol{A}$ during searching. If it is not found, it is searched in the current frame by the texture synthesis method [30].

\section{Experiments}

Experiments are conducted using a workstation with AMD Athlon (tm) II X4 640 3.01G, memory 2G, NVIDIA GT200 and CUDA 1.3. Two jittered videos are captured. The first is the video without moving object, and has 2476 frames, while the second is the video with moving object, and has 3124 frames.

As a special graphical processor, the computation performance and video memory bandwidth of GPU can be 10 times those of CPU. Therefore, the GPU+CPU framework [36] is used to achieve the real-time ability. Because the global motion estimation, motion filtering and compensation need large computation, they are implemented in GPU, while other parts are implemented in CPU. In addition, the GPU implement of SIFT [37] is used to accelerate the feature extraction. 

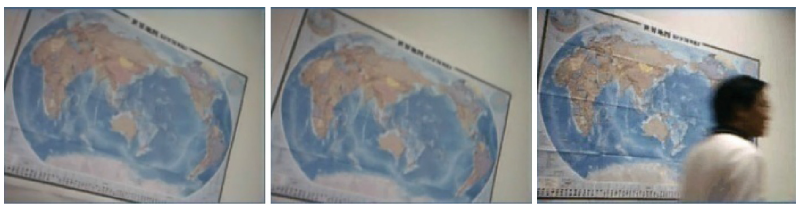

(a)
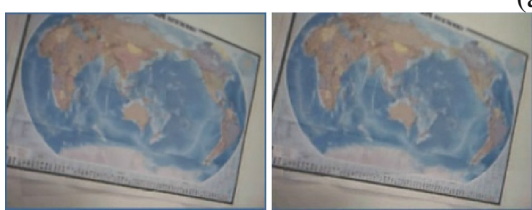

)

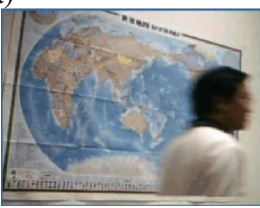

(b)
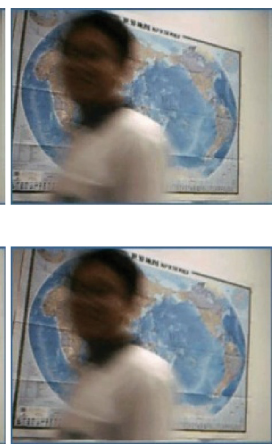

Fig. 2. (a) Current frames before video stabilization. (b) current frames after video stabilization.

Figure 2 shows the video stabilization results on the captured videos.

A video stabilization video can be downloaded from: http://staff.ustc.edu.cn/ $\sim$ harryjun/links/Video_Stabilization_1.avi. In addition, the real-time running process can be downloaded from: http://staff.ustc.edu.cn/ harryjun/links/Video_Stabilization_ 2.exe.

Figure 3 is the motion filtering results of $\boldsymbol{M}$ by EMD. They show the smoothing effect of EMD.

An index, peak signal-to-noise ratio (PSNR) between the reference frame $S_{0}$ and current frame $S_{1}$, is defined to evaluate the stabilization quality:

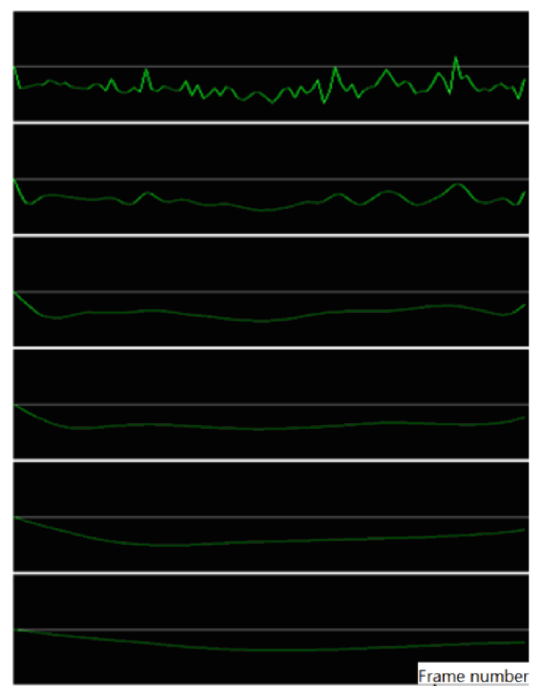

(a)

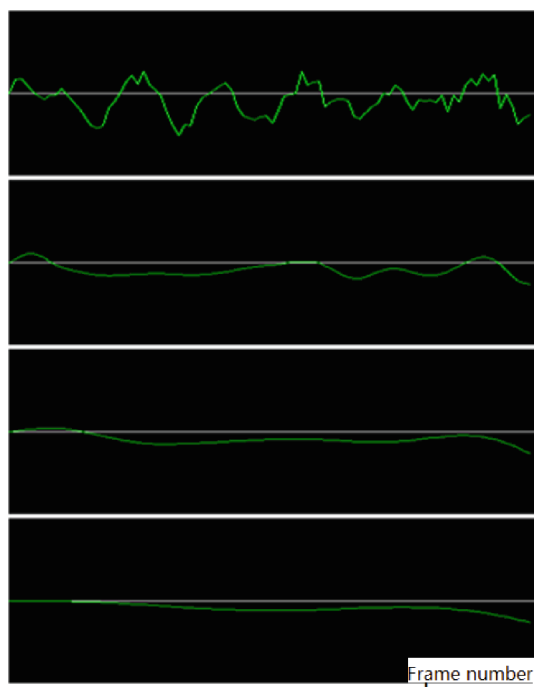

(b)

Fig. 3. The motion filtering results of $a_{3}$ : (a) and $a_{6}$ : (b) under different scales. 


$$
\operatorname{PSNR}\left(\boldsymbol{S}_{1}, \boldsymbol{S}_{0}\right)=10 \cdot \log _{10}^{255^{2} / \operatorname{MSE}\left(\boldsymbol{S}_{1}, \boldsymbol{S}_{0}\right)}
$$

Where $M S E$ is the mean square error of pixel value between two images. This index reflects the coherence between two images. The large the index, the better the video stabilization result.

Table 1 show the average PSNR on captured videos. As can be seen from it, the average $P S N R$ is increased by the proposed video stabilization method around $7.2 \mathrm{~dB}$, and the real-time ability is also achieved. Therefore, jittered video is stabilized by the proposed method nicely in real-time.

Table 1. Qualitative evaluation result of video stabilization.

\begin{tabular}{l|l|l|l}
\hline & $\begin{array}{l}\text { Average } P S N R \text { of } \\
\text { original videos }\end{array}$ & $\begin{array}{l}\text { Average } \text { PSNR of } \\
\text { stabilized videos }\end{array}$ & $\begin{array}{l}\text { Average time each } \\
\text { frame takes }\end{array}$ \\
\hline Captured video & 25.35 & 32.53 & $0.045 \mathrm{~s}$ \\
\hline
\end{tabular}

\subsection{Using Single Evaluation Criterion Vs. Fusing Multiple Evaluation Criteria}

The effect of fusing multiple evaluation criteria is verified on a video clip, in which some very frequent tiny rotation component is added. From Table 2, we can see the superiority of fusing multiple evaluation criteria.

Table 2. Evaluation between single evaluation criterion and multiple evaluation criteria.

\begin{tabular}{c|l|l|l}
\hline & $\begin{array}{l}\text { Average } P S N R \text { of } \\
\text { original videos }\end{array}$ & $\begin{array}{l}\text { Average } P S N R \text { of } \\
\text { stabilized videos }\end{array}$ & $\begin{array}{l}\text { Average time each } \\
\text { frame takes }\end{array}$ \\
\hline $\begin{array}{c}\text { Single evaluation } \\
\text { criterion }\end{array}$ & 18.56 & 24.04 & $0.037 \mathrm{~s}$ \\
\hline $\begin{array}{c}\text { Multiple evaluation } \\
\text { criteria }\end{array}$ & 18.56 & 24.79 & $0.045 \mathrm{~s}$ \\
\hline
\end{tabular}

\subsection{Objective Comparison with Other Algorithm}

The method in [29] is one of the state-of-the-art video stabilization methods. We have implemented it, then it and the proposed method are tested on the above video clip. We can see the proposed method is superior to the method in [29] from Table 3. This is because the proposed method fuses multiple evaluation criteria to conduct motion filtering by EMD, and stable SIFT features are used to match the adjacent frames robustly.

\subsection{Subjective Comparison with Other Algorithm}

The problem with an objective evaluation is that the absolute truth of camera motion is not known. However, it is less problematic for the subjective evaluation since the 
Table 3. Evaluation of several video stabilization algorithms.

\begin{tabular}{l|l|l|l}
\hline & $\begin{array}{l}\text { Average } P S N R \text { of } \\
\text { original videos }\end{array}$ & $\begin{array}{l}\text { Average PSNR of } \\
\text { stabilized videos }\end{array}$ & $\begin{array}{l}\text { Average time each } \\
\text { frame takes }\end{array}$ \\
\hline The proposed method & 18.56 & 24.79 & $0.045 \mathrm{~s}$ \\
\hline The method in [29] & 18.56 & 24.21 & $0.053 \mathrm{~s}$ \\
\hline
\end{tabular}

human visual system is very sensitive to the video jitter. The video stabilization system is developed as Fig. 4 shows. The original video is displayed in the upper-left window, while the stabilized video is displayed in the upper-right window. Afterwards, user's reactions interacting with this system are evaluated.

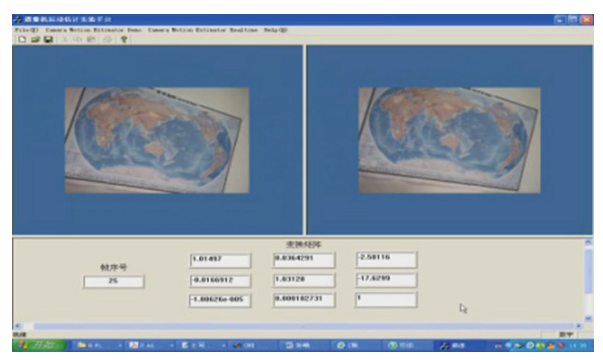

Fig. 4. Video stabilization system.

The participants in Table 4 are used. The goal of the evaluation is to decide if the system can remove the discomfort on human visual system, and if the objects in the stabilized video can be identified easily.

Table 4. Distributions of participants.

\begin{tabular}{l|l|l}
\hline Construct & Profiles & Distribution \\
\hline Age & $\begin{array}{l}\text { 1. Younger than 20. 2. Between 20 and 30. } \\
\text { 3. Older than 30. }\end{array}$ & $8 / 18 / 8$ \\
\hline Gender & 1. Male. 2. Female. & $20 / 14$ \\
\hline $\begin{array}{l}\text { Dialect } \\
\text { background }\end{array}$ & $\begin{array}{l}\text { 1. Northeastern. 2. Beijing. 3. Zhongyuan. 4. Southwestern. 5. } \\
\text { Xiang. 6. Gan. 7. Minnan. }\end{array}$ & $2 / 3 / 7 / 3 / 2 / 3 /$ \\
8. Cantonese. 9. Jilu. 10. Guiliu Pian. 11. Wu. & $2 / 4 / 3 / 1 / 4$ \\
\hline
\end{tabular}

In the first stage, the questionnaire is chosen for participants. Table 5 shows the constructs and questions of the survey related to the system performance. The answers to these questions are given from 'disagree' to 'agree' on a ten point scale. A Cronbach's alpha test [38] is carried out to determine if these constructs refer to the same topic. Typically, an alpha of 0.7 or greater is considered acceptable in psychological experiments. As Table 5 shows, all the alpha values obtained are greater than 0.7 , indicating that the questionnaire is suitable for the evaluation in this paper. 
Table 5. Cronbach's alpha results of questionnaire and mean scores after evaluation.

\begin{tabular}{l|l|l|l|l}
\hline Construct & Question & $\begin{array}{l}\text { Cronbach's } \\
\text { alpha }\end{array}$ & $\begin{array}{l}\text { Mean score of } \\
\text { the proposed } \\
\text { method }\end{array}$ & $\begin{array}{l}\text { Mean score } \\
\text { of the method } \\
\text { in [29] }\end{array}$ \\
\hline Smoothness & $\begin{array}{l}\text { If the stabilized video is } \\
\text { smooth and coherent. }\end{array}$ & 0.743 & 7.87 & 6.73 \\
\hline Identification & $\begin{array}{l}\text { If objects in the } \\
\text { stabilized video can } \\
\text { be identified easily. }\end{array}$ & 0.811 & 7.83 & 6.48 \\
\hline
\end{tabular}

In the second stage, the developed system and the method in [29] perform stabilization on captured videos, then participants compare stabilized videos with original videos. Finally, the questionnaire is filled. Table 5 shows the result of mean scores after evaluation. The maximum is 10 , while the minimum is 0 . For the developed system, all the scores obtained are greater than 7.5, and are higher than those of the method in [29], indicating that it has the ability to remove the discomfort on human visual system, and the objects in the stabilized video can be identified easily.

\section{Conclusion}

A real-time robust video stabilization system is proposed. The stable SIFT features are used to match the adjacent frames robustly. Global motion parameters are obtained by RANSAC to exclude wrong matches or correct matches that indeed belong to self-moving objects in the filmed scene effectively. Multiple evaluation criteria are fused to conduct motion filtering by EMD. The spatio-temporal information are combined with the texture synthesis method to obtain a complete video.

In future, the accuracy of global motion estimation will be further improved.

Acknowledgements. This work was supported by the National Natural Science Foundation of China (No. 61303150), the Open Project Program of the State Key Lab of CAD\&CG (No. A1501), Zhejiang University.

\section{References}

1. Ejaz, N., Wonil, K., Soon II, K., et al.: Video stabilization by detecting intentional and unintentional camera motions. In: International Conference on Intelligent Systems, Modelling and Simulation, pp. 312-316. IEEE Press, New York (2012)

2. Chen, C.H., Chen, C.Y., Chen, C.H., et al.: Real-time video stabilization based on vibration compensation by using feature block. Int. J. Innovative Comput. Inf. Control 7, 5285-5298 (2011)

3. Seok-Jae, K., Tae-Shick, W., Dae-Hwan, K., et al.: Video stabilization based on motion segmentation. In: IEEE International Conference on Consumer Electronics, pp. 416-417. IEEE Press, New York (2012) 
4. Dung, T.V., Lertrattanapanich, S., Cao, B.T., et al.: Real time video stabilization with reduced temporal mismatch and low frame buffer. In: IEEE International Conference on Consumer Electronics, pp. 61-62. IEEE Press, New York (2012)

5. Puglisi, G., Battiato, S.: A robust image alignment algorithm for video stabilization purposes. IEEE Trans. Circuits Syst. Video Technol. 21, 1390-1400 (2011)

6. Puglisi, G., Battiato, S.: Robust video stabilization approach based on a voting strategy. In: IEEE International Conference on Image Processing, pp. 629-632. IEEE Press, New York (2011)

7. Abraham, S.C., Thomas, M.R., Basheer, R., et al.: A novel approach for video stabilization. In: IEEE Recent Advances in Intelligent Computational Systems, vol. 1, pp. 134-137 (2011)

8. Uomori, K., Morimura, A., Ishii, H.: Electronic image stabilization system for video cameras and VCRs. J. Soc. Motion Picture Telev. Eng. 101, 66-75 (1992)

9. Pail, J.K., Park, Y.C., Kim, D.W.: An adaptive motion decision system for digital image stabilizer based on edge pattern matching. IEEE Trans. Consum. Electron. 38, 607-615 (1992)

10. Burt, A., Anandan, P.: Image stabilization by registration to a reference mosaic. In: Proceedings of ARPA Image Understanding Workshop, pp. 425-434. IEEE Press, New York (1994)

11. Morimoto, C., Chellappa, R.: Fast electronic digital image stabilization for off-road navigation. Real-Time Imaging 2, 285-296 (1996)

12. Ko, S.J., Lee, S.H., Lee, K.H.: Digital image stabilizing algorithms based on bit-plane matching. IEEE Trans. Consum. Electron. 44, 617-622 (1998)

13. Ko, S.J., Lee, S.H., Jeon, S.W., Kang, E.S.: Fast digital image stabilizer based on gray-coded bit-plane matching. IEEE Trans. Consum. Electron. 45, 598-603 (1999)

14. Erturk, S., Dennis, T.J.: Image sequence stabilization based on DFT filtering. IEE Proc. Image Vision Signal Proc. 127, 95-102 (2000)

15. Bosco, A., Bruna, A., Battiato, S., Bella, G.D.: Video stabilization through dynamic analysis of frames signatures. In: IEEE International Conference on Consumer Electronics, pp. 312 316. IEEE Press, New York (2006)

16. Censi, A., Fusiello, A., Roberto, V.: Image stabilization by features tracking. In: International Conference on Image Analysis and Processing, pp. 2354-2361. IEEE Press, New York (1999)

17. Veon, K.L., Mahoor, M.H., Voyles, R.M.: Video stabilization using SIFT-ME features and fuzzy clustering. In: IEEE/RSJ International Conference on Intelligent Robots and Systems, pp. 2377-2382. IEEE Press, New York (2011)

18. Windau, J., Itti, L.: Multilayer real-time video image stabilization. IEEE/RSJ International Conference on Intelligent Robots and Systems, pp. 2397-2402. IEEE Press, New York (2011)

19. Erturk, S.: Image sequence stabilization based on kalman filtering of frame positions. Electron. Lett. 37, 95-102 (2001)

20. Paik, P.: An adaptative motion decision system for digital image stabilizer based on edge pattern matching. In: Consumer Electronics, Digest of Technical Papers (1992)

21. Auberger, S., Miro, C.: Digital video stabilization architecture for low cost devices. In: Proceedings of the 4th International Symposium on Image and Signal Processing and Analysis, pp. 474-483. IEEE Press, New York (2005)

22. Tico, M., Vehvilainen, M.: Constraint translational and rotational motion filtering for video stabilization. In: Proceedings of the 13th European Signal Processing Conference, pp. 1474-1483. IEEE Press, New York (2005) 
23. Zhiyong, H., Fazhi H., Xiantao C., et al.: A 2D-3D hybrid approach to video stabilization. In: International Conference on Computer-Aided Design and Computer Graphics, pp. 146150. IEEE Press, New York (2011)

24. Litvin, A., Konrad, J., Karl, W.: Probabilistic video stabilization using kalman filtering and mosaicking. In: Proceedings of IS\&T/SPIE Symposium Electronic Imaging, Image, and Video Communications, pp. 663-674. IEEE Press, New York (2003)

25. Wexler, Y., Shechtman, E., Irani, M.: Space-time video completion. In: Proceedings of IEEE Conference on Computer Vision and Pattern Recognition, pp. 120-127. IEEE Press, New York (2004)

26. Jia, J., Wu, T., Tai, Y., Tang, C.: Video repairing: inference of foreground and background under severe occlusion. Proceedings of IEEE Conference on Computer Vision and Pattern Recognition, pp. 364-371. IEEE Press, New York (2004)

27. Cheung, S.C.S., Zhao, J., Venkatesh M.V.: Efficient object-based video in painting. In: IEEE International Conference on Image Processing, pp. 705-708. IEEE Press, New York (2006)

28. Cheung, V., Frey, B.J., Jojic, N.: Video Epitomes. In: Proceedings of IEEE Conference on Computer Vision and Pattern Recognition, pp. 42-49. IEEE Press, New York (2005)

29. Matsushita, Y., Ofek, E., Ge, W.N., et al.: Full-frame video stabilization with motion inpainting. IEEE Trans. Pattern Anal. Machine Intelligence. 28, 1150-1163 (2006)

30. Criminisi, A., Perez, P., Toyama, K.: Region filling and object removal by exemplar-based image inpainting. IEEE Trans. Image Process. 13, 1200-1212 (2004)

31. Tang, F., Ying, Y.T., Wang, J., et al.: A novel texture synthesis based algorithm for object removal in photographs. In: Proceedings of 9th Asian Computing Science Conference, pp. 248-258. IEEE Press, New York (2005)

32. Battiato, S., Gallo, G., Puglisi, G., Scellato, S.: SIFT features tracking for video stabilization. In: International Conference on Image Analysis and Processing, pp. 2255-2258. IEEE Press, New York (2007)

33. Lowe, D.: Distinctive image features from scale-invariant keypoints. Int. J. Comput. Vision 60, 91-110 (2004)

34. Hoper, P.J.: Robust statistical procedures. SIAM, Philadelphia (1996)

35. Huang, N.E., Shen, Z., Long, S.R., et al.: The empirical mode decomposition and the Hilbert spectrum for nun-linear and non-stationary time series analysis. Proc. R. Soc. Lond. A 454, 903-995 (1998)

36. Juang, C., Chen, T., Cheng, W.: Speedup of implementing fuzzy neural networks with high-dimensional inputs through parallel processing on graphic processing units. IEEE Trans. Fuzzy Syst. 19, 717-728 (2011)

37. http://cs.unc.edu/ ccwu/siftgpu/

38. Marcosa, S., Gómez-García-Bermejob, J., Zalama, E.: A realistic, virtual head for human-computer interaction. Interact. Comput. 22, 176-192 (2010) 